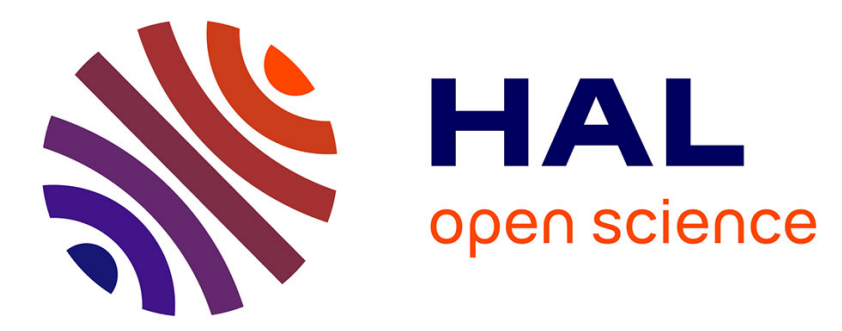

\title{
Transport properties of catalyst supports studied by pulsed field gradient (PFG) and 2D exchange (EXSY) NMR spectroscopy
}

\author{
Erika Weiland, Marie-Anne Springuel-Huet, Andrei Nossov, Flavien \\ Guenneau, Anne-Agathe Quoinneaud, Antoine Gedeon
}

\section{To cite this version:}

Erika Weiland, Marie-Anne Springuel-Huet, Andrei Nossov, Flavien Guenneau, Anne-Agathe Quoinneaud, et al.. Transport properties of catalyst supports studied by pulsed field gradient (PFG) and 2D exchange (EXSY) NMR spectroscopy. New Journal of Chemistry, 2016, 40 (5), pp.4447-4454. 10.1039/C5NJ02952J . hal-01331111

\section{HAL Id: hal-01331111 \\ https://hal.sorbonne-universite.fr/hal-01331111}

Submitted on 13 Jun 2016

HAL is a multi-disciplinary open access archive for the deposit and dissemination of scientific research documents, whether they are published or not. The documents may come from teaching and research institutions in France or abroad, or from public or private research centers.
L'archive ouverte pluridisciplinaire HAL, est destinée au dépôt et à la diffusion de documents scientifiques de niveau recherche, publiés ou non, émanant des établissements d'enseignement et de recherche français ou étrangers, des laboratoires publics ou privés. 


\title{
Journal Name
}

\section{Transport properties of catalyst supports studied by pulsed field gradient (PFG) and 2D exchange (EXSY) NMR spectroscopy}

\author{
Erika Weiland, ${ }^{a, b}$ Marie-Anne Springuel-Huet ${ }^{b *}$, Andrei Nossov ${ }^{b}$, Flavien Guenneau ${ }^{b}$, Anne-Agathe \\ Quoinneaud $^{\mathrm{a}}$ and Antoine Gédéon ${ }^{\mathrm{b}^{*}}$

\begin{abstract}
Self-diffusivity of xenon and $n$-hexane in alumina supports have been measured by ${ }^{129} \mathrm{Xe}$ and ${ }^{1} \mathrm{H}$ PFG NMR. The values were used to evaluate the tortuosity of the complexe porosity of aluminas. The pore connectivity has been investigated by ${ }^{129} \mathrm{Xe}$ 2D EXSY NMR. The exchange rates of xenon atoms between different pore domains or between adsorbed and gas phase have been determined using a two site exchange model. The exchange rate together with the diffusivity values allowed evaluating a mean size of the pore domains.
\end{abstract}

\section{Introduction}

Energy production, which is one of the most crucial issues in the world, is intimately linked to economic activity. The most widely used sources of energy are the fossil fuels which contain sulfur compounds. These latter are an environmental concern because upon combustion sulfur are converted to SOx, chemical compounds which not only have negative effect on human health and in the environment, but also tend to deactivate catalysts used in crude oil processing.

Interest in the hydrodesulfurization (HDS) of diesel fractions has been revived by specifications concerning the sulfur content of diesel fuel. Since 2009 , the maximum sulfur content of diesel fuel has been limited to $10 \mathrm{ppm}$ in most parts of the industrial world. Many studies have been performed to develop new catalysts particularly active in the HDS of refractory molecules. These catalysts are also designed to improve the existing refining processes in order to convert heavy distillates to more valuable light ones used in petrochemistry and transport. Although sulfidation of the supported oxidic Mo, CoMo and NiMo catalysts ${ }^{1-3}$ has received considerable attention; more detailed knowledge about their

\footnotetext{
a. IFP Energies nouvelles, Rond-point de l'échangeur de Solaize- BP3, -69360 Solaize, France.

b. Sorbonne Universités, UPMC Univ Paris 06, CNRS-UMR 7574, Laboratoire de Chimie de la Matière Condensée de Paris, 11 place Marcelin Berthelot, F-75005, Paris, France.
}

Electronic Supplementary Information (ESI) available: T1 relaxation times of $n$ hexane and xenon in Al_237_m, Al_204_m, Al_224_b and in bulk phases. PFG attenuation curves of liquid $n$-hexane impregnated in Al_224-b and Al_204_m, of xenon in Al_224_b, of $n$-hexane adsorbed in Al_237_m, Al_204_m and Al_224_b. $1 \mathrm{D}{ }^{129}$ Xe spectrum of Al 224 b. Variations of the diffusivity of liquid and adsorbed $\mathrm{n}$-hexane with the observation times for the three aluminas. Tortuosity of the three aluminas determined from diffusivity of adsorbed $n$-hexane. See DOI: $10.1039 / x 0 x \times 00000 x$ preparation and interaction chemistry is desirable. Owing to their unique properties such as their high specific surface area, mechanical, chemical and thermal stability, transition aluminas are at the heart of key applications in numerous industrial hydrotreatment processes. ${ }^{4,5}$

Understanding the transport behaviour of molecules is important for refining processes and other industrial applications such as separation, adsorption. Diffusion of molecules in porous media can be measured by a large variety of experimental methods, leading to a wide range of diffusion coefficients (Figure 1). Pulsed field gradient (PFG) NMR has proved to be a powerful technique to investigate diffusion phenomena inside nanopores. ${ }^{6}$ It is able to follow molecular displacements from some hundreds of nanometers up to hundreds of micrometers. This technique has proved to be particularly suited for diffusion studies within microporous ${ }^{7,8}$ mesoporous $^{9,10}$ or also in more complex porous materials, presenting both micro- and mesoporosity, ${ }^{11}$ or consisting of zeolites intergrowth. ${ }^{12}$ Recently, ${ }^{1} \mathrm{H}$ PFG-NMR allowed distinguishing true hierarchical materials from a mechanical mixture made of independent micro- and mesoporous materials. $^{13}$

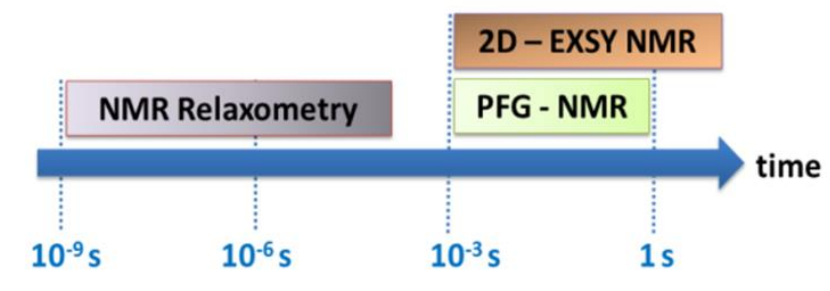

Figure 1. Time scale of some NMR techniques 
The transport properties of catalysts can be characterized by the tortuosity factor, which provides a means of quantifying the extent of increased molecular trajectories in porous systems due to multiple reflections against the pore walls. The determination of the tortuosity provides a good understanding of molecular diffusion and the complex porous system. The tortuosity factor, $\eta$, is defined as the ratio of $L_{\text {eff }} / L$, where $L_{\text {eff }}$ is the effective path length of a molecule crossing a porous bed of width L. ${ }^{14}$ However, the complex nature of porous systems makes it difficult to quantify tortuosity. The tortuosity factor, can also be determined from the self-diffusivity (D) of a molecule diffusing in the pore systems. ${ }^{15,16}$ Actually, the tortuosity can be defined by the ratio $D_{0} / D$ where $D_{0}$ is the reference self-diffusivity, usually defined as the diffusivity of the same molecules under the same conditions of temperature and pressure in a single cylinder of infinite length with diameter equal to the mean intercept of the pore structure considered.

In materials presenting hierarchical porosity, the extent of connectivity between nano- or mesopore domains markedly influences transport properties of the materials. In nanoporous materials, both pore size and interconnectivity greatly affect the molecular transport properties. The pore geometry in most porous materials, even in ordered silica, is complex and characterized by interconnected pores. As a result of such complex topography, the characterization of pore connectivity in nano- or mesoporous materials is often challenging and mandates several methods. ${ }^{129} \mathrm{Xe}$ NMR of adsorbed xenon can provide information about the pore space and transportation processes in porous materials. Since its first applications to the study of microporous solids, ${ }^{129}$ Xe NMR has become a very popular technique for the study of zeolites and other porous materials. Very recently, a correlation between the ${ }^{129}$ Xe chemical shift and the pore size was established for alumina supports. ${ }^{17}$ There are several reviews on the applications of xenon NMR to microporous materials. ${ }^{18-20}$

In contrast to the most common techniques such as smallangle X-ray or neutron scattering, and gas adsorption, 2D exchange spectroscopy (EXSY) ${ }^{129}$ Xe NMR can provide direct information on how pores are connected and, therefore, a better understanding of the pore structure, the uniformity of the adsorption sites and the pore connectivity between different adsorption regions. ${ }^{21}$

The objective of this work is to investigate the transport properties of alumina materials using ${ }^{1} \mathrm{H}$ PFG NMR as well as the pore connectivity by $2 \mathrm{D}$ EXSY- ${ }^{129}$ Xe NMR techniques.

\section{Experimental}

\section{Materials, characterization and sample preparation}

The $\gamma$-alumina supports were obtained by precipitation in aqueous solution of aluminum salts. The boehmite precipitate was filtered and washed to minimize the quantity of sulfate or sodium ions depending on aluminum salt solution. Shaping involves the passage from the boehmite powder to support pellets. The extrudates are cylindrical or trilobal. Their diameter ranges from 1.2 to $2 \mathrm{~mm}$ and the length from 2 to
$6 \mathrm{~mm}$. A thermal treatment at high temperature (from $798 \mathrm{~K}$ to $1248 \mathrm{~K}$ ) was performed to obtain the final support.

The specific surface area $(\mathrm{S})$, pore volume $(\mathrm{V})$ and the pore diameter (d) of aluminas were determined from nitrogen sorption isotherm at $77 \mathrm{~K}$ measured with a BelsorpMax (Bel Japan, Inc.) porosimeter. Prior to analysis, the samples were pretreated at $573 \mathrm{~K}$ overnight with the same protocol as that used before NMR experiments (see below). S and $\mathrm{V}$ were determined using the BET equation and $\mathrm{BJH}$ analysis (cylindrical pore model), respectively. The total mesopore volume (V) was determined from the amount of $\mathrm{N}_{2}$ adsorbed at $P / P_{0}=0.99$. The $t$-plot analyses do not show any presence of micropores.

About $300 \mathrm{mg}$ of alumina extrudates were placed in a NMR tube provided with a valve. The samples were dehydrated under dynamic vacuum (less than $10^{-1} \mathrm{~Pa}$ ) at room temperature for $30 \mathrm{~min}$ and then at $573 \mathrm{~K}$ for $17 \mathrm{~h}$ (heating rate $100 \mathrm{~K} / \mathrm{h}$ ). Then, the extrudates were immersed in liquid $\mathrm{n}$ hexane (Fluka 99\%) inside a glovebox for three days. Then, they were exposed to air, their external surface was dried with absorbing paper and they were introduced into $10 \mathrm{~mm}$ od NMR tube. A piece of absorbing paper wetted with a small amount of $n$-hexane was introduced at the top of the tube to prevent evaporation of $n$-hexane from alumina.

For the experiments with xenon, the treated samples were subjected to a pressure of $1 \mathrm{MPa}$ of xenon (Air Liquide) obtained by condensing a calibrated amount of xenon in the NMR tube which was subsequently sealed.

\section{PFG NMR experiments}

The PFG experiments were run on a Bruker Avance 300 spectrometer operating at $300.16 \mathrm{MHz}$ for ${ }^{1} \mathrm{H}$ and $83.03 \mathrm{MHz}$ for ${ }^{129} \mathrm{Xe}$. It was equipped with a Diff50 probe delivering a maximum gradient of $30 \mathrm{~T} \mathrm{~m}^{-1}$. Whenever possible, the bipolar 13-interval pulse sequence ${ }^{22}$ (PGSTEBP) was chosen to avoid any effect arising from the presence of internal field gradients in the materials. When the relaxation times (see Table S1 in ESI) were too short, a simple pulsed gradient spin echo (PGSE) sequence was used. ${ }^{23}$ For the PGSTEBP sequence, the echo attenuation, $\Psi=1 / I_{0}$, takes the form (adapted from reference 2).

$$
\Psi(g, \delta, \Delta)=\exp \left(-(\gamma \delta \mathrm{g})^{2} \mathrm{D}\left(\Delta-\frac{\tau}{2}-\frac{\delta}{6}\right)\right) \quad \text { Equation } 1
$$

where $D$ is the diffusion coefficient. $\Delta$ is the observation time, i.e. the time interval between the gradient pulses. $\gamma$ is the gyromagnetic ratio; $\delta$ and $g$ denote the duration and the strength of the gradient pulses, respectively. $\tau$ is the time interval between the last radio-frequency pulse and the spin echo (echo time). For the PGSE experiments, the last parameter in the exponential of equation 1 is reduced to $\Delta-\delta / 3$. The gradient pulse duration, $\delta$, was set to $1 \mathrm{~ms}$ and the echo time $\tau$ to $5.6 \mathrm{~ms}$. The observation time was varied from 7 to $300 \mathrm{~ms}$ at maximum. The intensity of the applied gradients was set between 0 and $2.5 \mathrm{~T} \mathrm{~m}^{-1}$ for $\mathrm{n}$-hexane experiments and between 0 and $1.0 \mathrm{~T} \mathrm{~m}^{-1}$ for xenon experiments. 


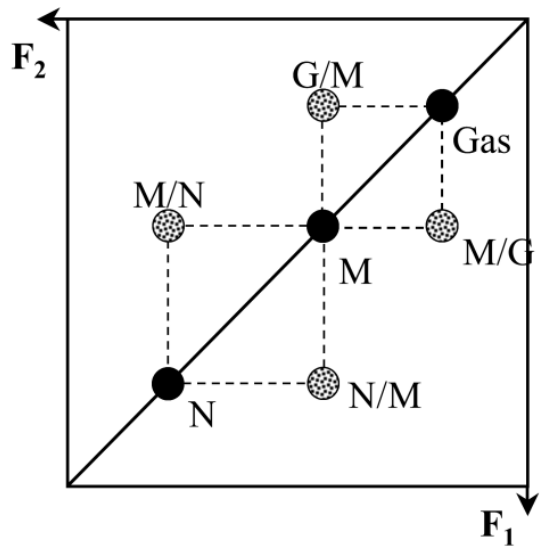

Figure 2. Schematic 2D map showing exchange of xenon between the gas phase (G) and the porosity $M$ on one hand and between the porosity $M$ and the porosity $\mathrm{N}$, on the other hand (no exchange between $\mathrm{G}$ and $\mathrm{N}$ ).

The duration of the $\pi / 2$ radiofrequency pulse was $20 \mu$ s and 11 $\mu$ s for ${ }^{1} \mathrm{H}$ and ${ }^{129} \mathrm{Xe}$, respectively.

All the experiments were performed at $298 \mathrm{~K}$. The selfdiffusivity, $D$, is obtained by fitting the echo attenuation curves In $\Psi=f\left(g^{2}\right)$ to equation 1 . The uncertainty on the $D$ values given below corresponds to two times the value of the standard deviation of the $\mathrm{D}$ values obtained at different observation times.

\section{${ }^{129}$ Xe 2D EXSY NMR experiments}

${ }^{129}$ Xe 2D EXSY NMR experiments have been performed using a $\pi / 2-t_{1}-\pi / 2-t_{m}-\pi / 2-t_{2}$ pulse sequence. The $2 D$ spectra were acquired with a spectral width of $15 \mathrm{kHz} .64$ and 1024 points were acquired for the $t_{1}$ and $t_{2}$ dimensions, respectively and the mixing time, $t_{m}$, was varied from 0.5 to $400 \mathrm{~ms}$. Before Fourier transformation, the $t_{1}$ dimension was zero filled to 1024 points. 2D EXSY NMR correlates the frequencies of the ${ }^{129}$ Xe spins in the evolution $\left(t_{1}\right)$ and detection $\left(t_{2}\right)$ periods. Changes in the NMR frequencies during the mixing time $\left(t_{m}\right)$ result in off-diagonal intensities in the $2 \mathrm{D}$ spectrum as schematically shown in Figure 2 . The $1 \mathrm{D}$ spectrum is found in the diagonal.

\section{Results and discussion}

The calcination of boehmite giving rise to alumina is a topotactic transformation. ${ }^{24,25}$ Therefore, the nanosized platelet crystals of boehmite are found in aluminas. The complex porosity of the latter originates from the disordered aggregation of the platelets and the agglomeration of aggregates. ${ }^{26} \mathrm{~N}_{2}$ sorption experiments at $77 \mathrm{~K}$ reveal that the porosity is monomodal or bimodal (based on $\mathrm{BJH}$ analysis). The textural properties of the three aluminas obtained from $\mathrm{N}_{2}$ sorption are given in Table 1 . The samples are denoted Al_X_m(or $b$ ), where $X$ is the BET surface area and $m$ or $b$ indicates a monomodal or bimodal porosity.
Self-diffusivity of liquid $\mathrm{n}$-hexane in alumina supports measured by ${ }^{1} \mathrm{H}$ PFG NMR.

The self-diffusivity of liquid $\mathrm{n}$-hexane impregnated in the three alumina samples has been measured by ${ }^{1} \mathrm{H}$ PFG NMR.

Table 1. Specific surface areas, pore volumes and mean pore sizes determined from $\mathrm{N}_{2}$ sorption at $77 \mathrm{~K}$.

$\begin{array}{cccc}\text { sample } & \text { S (BET) m2g-1 } & \text { V pore cm3g-1 } & \text { d nm } \\ \text { Al_224_b } & 224 & 0.55 & 5.7 ; 9.9 \\ \text { Al_237_m } & 237 & 0.64 & 8.9 \\ \text { Al_204_m } & 204 & 0.62 & 10.9\end{array}$

The logarithm of the echo signal attenuation, $1 / I_{0}$, versus the square of the field gradient, $\mathrm{g}^{2}$, obtained for Al_237_m is shown in Figure 3. The fit of the experimental data to equation 1 gives a diffusivity value, $D$, which is independent of the observation time, $\Delta$ (between $7 \mathrm{~ms}$ to $300 \mathrm{~ms}$ ). It is also the case for Al_204_m and Al_224_b (see ESI, Figures S1, S2, S3). D values are given in Table 2 .

One can notice that during the observation time used (between 7 and $300 \mathrm{~ms}$ ), the root mean square displacement (rms), $V\left\langle R^{2}\right\rangle$, of the diffusing molecules, calculated with the Einstein equation $\left(\left\langle R^{2}\right\rangle=6 D \Delta\right)$ is between $8-9$ and $50-58$ microns depending on the aluminas. This length corresponds roughly to the size of the agglomerates constituting the alumina extrudates. The independency of $\mathrm{D}$ with $\Delta$ means that the $\mathrm{n}$-hexane molecules do not encounter diffusion barriers at the agglomerate scale. ${ }^{27}$ In addition, these rms values are smaller than the size of the domains of distinct pore sizes detected in Al_224_b by ${ }^{129} \mathrm{Xe}$ NMR thanks to the high sensitivity of the Xe atoms to their environment (vide infra). Most of $n$-hexane molecules diffuse within one domain only. Within the range of gradient strength used, all attenuation curves can be fitted with a mono-exponential, therefore the existence of two different diffusivities corresponding to the two domains (characterized by their pore size) cannot be stated.

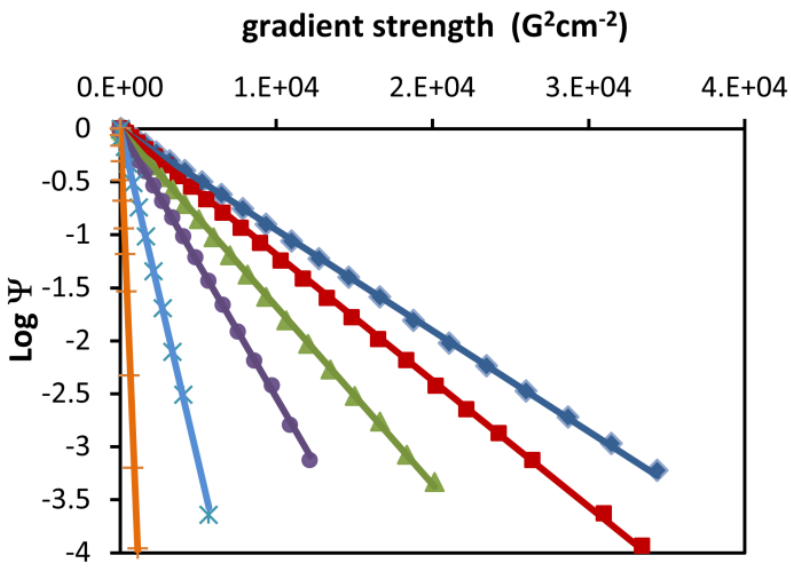

Figure 3. Variation of $\ln \Psi$ versus the square of the field gradient $\left(\mathrm{g}^{2}\right)$ of liquid $\mathrm{n}$ hexane impregnated in Al_237_m for some observation times, $\Delta: 7.5 \mathrm{~ms}$ (blue lozenges), $10 \mathrm{~ms}$ (red squares), $14 \mathrm{~ms}$ (green triangles), $20 \mathrm{~ms}$ (purple circles), 50 $\mathrm{ms}$ (light blue stars), $300 \mathrm{~ms}$ (orange crosses). The solid lines represent the least square fits to the data. 
It can also be noticed that the fraction of molecules interacting with the surface, whose diffusivity should be smaller than that of the molecules in the volume of the pore, is not highlighted by a change of slope of the attenuation curves as it was the case for 1 -octene diffusing in $\mathrm{Pd} / \theta$-alumina catalyst. ${ }^{28}$ One reason may be the gradients used which are not large enough to "pick up" slow molecules. In addition, a rapid calculation shows that the fraction of these molecules is about $8-10 \%$ only assuming a monolayer of molecules on the surface.

The knowledge of diffusivities of molecules diffusing in mesopores allows determining the tortuosity $(\eta)$ of materials. It represents the effect of confinement: pore orientation, connectivity, size variation and all the factors which may constitute a resistance to the movement of molecules inside the materials. There are different theoretical approaches proposed to evaluate this physical quantity. When considering the effective diffusivities of the diffusing molecules, the tortuosity, $\eta$, has been defined by the ratio, $D_{0} / D$ eventually corrected by the porosity of the porous medium when macroscopic measurements are used. ${ }^{16}$ In the present PFG NMR study of aluminas presenting rather large pores compared to the size of diffusing molecules and impregnated with liquid-hexane, the diffusion regime is what is called "the bulk regime". It means that molecule-molecule collisions dominate the diffusion process in contrary to the "Knudsen regime" for which the molecule-solid collisions govern the diffusion. ${ }^{29}$ In the present case (bulk regime), the tortuosity, $\eta_{B}$, can be determined by the ratio of the diffusivity, $D_{0}$, of molecules in the pure liquid $n$-hexane and the diffusivity, $D$, of $\mathrm{n}$-hexane molecules inside the pores. ${ }^{30}$

$$
\eta_{\mathrm{B}}=\frac{\mathrm{D}_{0, \mathrm{~B}}}{\mathrm{D}_{B}}
$$

Equation 2

Tortuosity values obtained for the three alumina samples are given in Table 2.

These values are in good agreement with those published in the literature for $\gamma$-alumina; for example D'Agostino et al. obtained a value of $1.7 .^{31}$

As mentioned above, in case of alumina with bimodal porosity ${ }^{129}$ Xe NMR spectroscopy is able to differentiate the two types of pores and the presence of the corresponding distinct signals can be used to determine the xenon diffusivity within the two types of pores of an alumina. This is not possible with ${ }^{1} \mathrm{H} N M R$ due to the very narrow chemical shift range compared to that of ${ }^{129} \mathrm{Xe}$.

\section{Self-diffusivity of xenon in alumina supports measured by ${ }^{129} \mathrm{Xe}$ PFG NMR}

${ }^{129} \mathrm{Xe}$ NMR is much less sensitive than ${ }^{1} \mathrm{H}$ NMR spectroscopy. In the aim to obtain a sufficient signal-to-noise ratio the samples were subjected to a xenon pressure of 10 bars. Nevertheless, it was not possible to detect an echo signal for Al_237_m neither for Al_224_b with the long PGSTEBP sequence due to the short $\mathrm{T}_{2}$ relaxation times of xenon adsorbed in these aluminas (see ESI, Table S1). However, with the short PGSE sequence an echo signal was detected for Al_224_b but not for Al_237_m (due to too short $\mathrm{T} 2$ relaxation time).
Figure 4 shows the echo signal attenuation, $I / I_{0}$, versus the square of the field gradient, $\mathrm{g}^{2}$, obtained for xenon adsorbed in Al_204_m. using the PGSTEBP sequence. The fit of this variation with equation 1 gives a diffusivity of $6.2 \times 10^{-8} \mathrm{~m}^{2} \mathrm{~s}^{-1}$. For Al_224_b, the ${ }^{129}$ Xe NMR spectrum shows two signals corresponding to the two types of porosities of this alumina (Figure S4, ESI section). Then, it was possible to determine two different diffusivities, namely $2 \times 10^{-8}$ and $3.8 \times 10^{-8} \mathrm{~m}^{2} \mathrm{~s}^{-1}$, associated to the two types of pores (5.7 and $9.9 \mathrm{~nm}$ ), respectively (Table 2 and Figure S5, ESI section). The diffusion of xenon is faster in the larger pores. As mentioned above, it was not possible to figure out two diffusivities for liquid nhexane in Al_224_b. One reason is that, in bulk regime, the diffusivity of molecules should not depend on the pore size in contrary of the diffusivity in Knudsen regime which is proportional to the pore size (vide infra).

Note that it is difficult to compare the diffusivity of xenon adsorbed in Al_204_m and in Al_224_b since they are obtained using different NMR pulse sequences. The PGSTEBP sequence used with Al_204_m allows minimizing the effect of internal field gradient and eddy currents on the diffusivity measurements. To evaluate their effect, the diffusivity of xenon in Al_204_m was also measured using the simple PGSE sequence. A value of $4.9 \times 10^{-8} \mathrm{~m}^{2} \mathrm{~s}^{-1}$ was obtained which is about $25 \%$ undervalued with respect to the value obtained with the PGSTEBP sequence. Even if we assume that the xenon diffusivities obtained for Al_224_b are similarly undervalued, they remain smaller than that obtained for Al_204_m. Therefore, it can be observed that the diffusivity of liquid $n$ hexane and adsorbed xenon varies in the opposite order for the three aluminas.

One must keep in mind that the diffusivity measured by PFG NMR represents the diffusivity of all the molecules inside the pore, that is the molecules adsorbed on the pore surface and the "free" molecules in the pore volume.

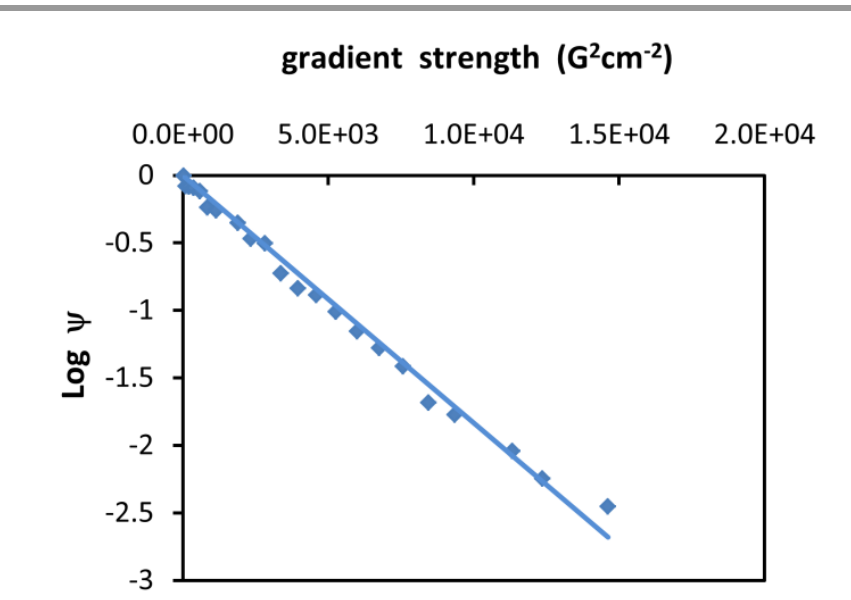

Figure 4. Variations of $\ln \psi$ versus the square of the field gradient (g2) of xenon adsorbed under $1 \mathrm{MPa}$ in Al_204_m for $\Delta=8 \mathrm{~ms}$. The solid line represents the least square fit to the data. 
In case of experiments with aluminas impregnated with liquid n-hexane, the fraction of molecules interacting with the surface (assuming a monolayer of adsorbed molecules) is around $10 \%$.

This can be considered as negligible because the surface diffusivity is expected to be smaller than the "bulk" diffusivity. For the experiments with adsorbed xenon, if we assume that xenon adsorption follows a Henry's law at pressures up to 10 bars, the fraction of xenon atoms adsorbed on the surface is estimated around $80-90 \%$. This value is evidently largely overstated (because of the Henry's law assumption) but it shows that most of xenon atoms inside the pores are adsorbed on the surface. Nevertheless, their influence on the measured diffusivity might be negligible if the surface diffusion is several orders of magnitude smaller than the diffusivity of the free atoms in the volume (which can be considered as "Knudsen" diffusivity).

If we consider that the diffusivity of xenon inside the pores is essentially due to the collisions with the solid, a tortuosity factor can also be derived in the same way as for liquid nhexane (see equation 2) from xenon diffusivity. In that case, the reference diffusivity, $D_{0, k}$, must be the diffusivity of a molecule diffusing in a cylinder of infinite length with diameter equal to the mean intercept of the pore structure considered. This theoretical quantity can be calculated: ${ }^{16}$

$$
D_{0, K}=97 r \sqrt{T / M}
$$

Equation 3

where $r, T$ and $M$ are the pore radius, the temperature and the molecular weight of the diffusing molecule, respectively.

The obtained tortuosities, $\eta_{\mathrm{K}}$, are given in Table 2 .

The values of $\eta_{\mathrm{K}}$ are significantly larger than those obtained in the bulk regime with liquid $n$-hexane $(\eta)$. This discrepancy between the tortuosity values determined in bulk and Knudsen regimes has already been observed from both experimental and Monte Carlo simulation experiments. ${ }^{32-34}$ This finding has been tentatively attributed to specific interactions of the diffusing molecule with particular sites (strong adsorption sites) on the pore surface. ${ }^{33}$ In our experimental conditions, the majority of the surface is covered with xenon atoms and these sites must be masked to the "free" xenon atoms in the pore volume.

On the other hand, the assumption of a pure Knudsen regime might be questionable. The mean free path, $\ell$, of a xenon atom in a pore can be estimated. $\ell$ is the difference between the pore diameter/radius and the atom diameter/radius in an infinite cylinder/closed sphere. ${ }^{35}$

Table 2. Diffusivity of liquid $n$-hexane and adsorbed xenon ( $P=10$ bar) in alumina samples and their tortuosity.

$\begin{array}{lllll}\text { sample } & \mathrm{D}_{\mathrm{C} 6 \mathrm{H} 14}\left(\mathrm{~m}^{2} \mathrm{~s}^{-1}\right) & \eta^{\text {(a) }} & \mathrm{D}_{\text {xe }}\left(\mathrm{m}^{2} \mathrm{~s}^{-1}\right) & \eta^{\text {(b) }} \\ \text { Al_224_b } & 1.9 \times 10^{-9} & 1.4 & 2 \times 10^{-8}(5.7 \mathrm{~nm}) & 17 \\ & & & 3.8 \times 10^{-8}(9.9 \mathrm{~nm}) & 15 \\ \text { Al_237_m } & 1.8 \times 10^{-9} & 1.5 & - & - \\ \text { Al_204_m } & 1.4 \times 10^{-9} & 1.9 & 6.2 \times 10^{-8} & 13\end{array}$

(a) determined from $D_{\mathrm{C} 6 \mathrm{H} 14}$ and $\mathrm{D}_{0, \mathrm{~B}}=2.7 \times 10^{-9} \mathrm{~m}^{2} \mathrm{~s}^{-1}$ (value measured by PFG NMR on pure liquid $\mathrm{n}$-hexane at $298 \mathrm{~K}$ ).

(b) determined from $D_{x e}$ and $D_{0, k}$ calculated with equation 3 for each pore radius.
The pore geometry of the alumina samples is not known but is something between these two geometries. So we can estimate the value of $\ell$ between 2.2 and $4.4 \mathrm{~nm}$ for the smallest pores $\left(D_{\mathrm{BJH}}=5.7 \mathrm{~nm}\right)$ and between 4.8 and $9.6 \mathrm{~nm}$ for the largest ones $\left(D_{\text {BJH }}=10.9 \mathrm{~nm}\right)$. These values have to be compared to the mean free path of the xenon atoms (around $4.8 \mathrm{~nm}$ ) in gas phase under 10 bars. Therefore, it cannot be excluded that molecule-molecule collisions are not involved in the diffusion processes of xenon inside the pores. On can notice that this can be an additional reason to explain why the diffusivity/tortuosity, measured with liquid $n$-hexane and adsorbed xenon, varies in the opposite way.

In order to work in Knudsen regime with an adsorbate at low pressure to minimize molecule-molecule collisions, we measured the diffusivity of adsorbed $n$-hexane. We performed PFG NMR experiments on the three aluminas with gaseous $n-$ hexane under a pressure of $6 \mathrm{kPa}$ (Figures S6 and S7, ESI section). At this pressure, the mean free path of a molecule in the gas phase is $144 \mathrm{~nm}$ that is much larger than the mean free path of a molecule diffusing inside the pores of aluminas. The diffusivities obtained under these conditions are $1.5 \times 10^{-9}$, $1.9 \times 10^{-9}$ and $2.5 \times 10^{-9} \mathrm{~m}^{2} \mathrm{~s}^{-1}$ for Al_224_b, Al_237_m and Al_204_m, respectively. The values are also independent of the observation time (Figure S8, ESI section). The tortuosities determined by using the calculated Knudsen diffusivity, as we have done for ${ }^{129}$ Xe experiments, are 468 (average on the two types of pores), 422 and 393, respectively. These values are extremely high and not representative of the real tortuosity of the materials because, under the experimental conditions, the influence of the adsorbed molecules is predominant in the measured diffusivity. In fact, the fraction of adsorbed molecules is about 1000 and therefore, the measured diffusivity is governed by the surface diffusion processes.

To better characterize the textural properties of the aluminas we performed 2D EXSY ${ }^{129}$ Xe NMR experiments.

\section{Exchange of xenon between different porosities or between porosity and gas phase measured by ${ }^{129}$ Xe 2D EXSY NMR.}

${ }^{129}$ Xe NMR is known to be a useful technique to characterize materials porosity. ${ }^{18,19}$ In particular, the chemical shift of xenon adsorbed in porous solids has been correlated to the mean pore size for zeolites and related materials as well as for mesoporous silicas. ${ }^{35-37}$ Recently, we established a new correlation adapted to transition aluminas. ${ }^{17}$

When the exchange of xenon atoms between different types of environments is not too fast (exchange rate smaller than the frequency difference between the NMR signals characteristic of each environment), the spectra show as many signals as there are types of environments. ${ }^{38}$ As shown above, it is the case of Al_224_b alumina which presents a bimodal porosity. In addition to the gas phase signal (noted $G$ ), the 1D spectrum shows two signals (noted $A$ and $B$ ) corresponding to the two types of pores, separated by 10 ppm, i.e. $830 \mathrm{~Hz}$ (see Figure S2).

The observation of these two signals means that the exchange rate of xenon atoms between the two porosities $A$ and $B$ is 
smaller than $830 \mathrm{~Hz}$. It is possible to investigate the exchange at smaller rate using 2D EXSY experiments. In such experiments, the spins may change their environment, therefore their resonance frequency, during a so-called "mixing time, $\mathrm{t}_{\mathrm{m}}$ " giving a cross peak in the 2D EXSY spectrum. Figure 5 shows the ${ }^{129}$ Xe 2D EXSY spectrum of xenon adsorbed in Al_224_b for a mixing time of $50 \mathrm{~ms}$.

Cross-peaks between $A$ and $B$ signals as well as between $A$ and $G$ ones are observed.

To determine the exchange rate, $k$, between the porosities $A$ and $B$, we considered that the porosity $B$ does not exchange with the gas phase and is involved in a two-site exchange only. Therefore, we used the expressions of the diagonal peak $\left(\mathrm{I}_{\mathrm{BB}}\right)$ and cross-peak $\left(\mathrm{I}_{\mathrm{AB}}\right)$ intensities derived by Jeener ${ }^{39}$ for a twosite exchange model. The ratio $\mathrm{I}_{\mathrm{AB}} / \mathrm{I}_{\mathrm{BB}}$ can be written as:

$$
\frac{I_{A B}}{I_{B B}}=\frac{x_{A} k \sinh \left(\alpha t_{m}\right)}{\alpha\left[\cosh \left(\alpha t_{m}\right)-\frac{\beta}{\alpha} \sinh \left(\alpha t_{m}\right)\right]}
$$

Equation 3

With $\alpha=\sqrt{\beta^{2}+x_{A} x_{B} k^{2}}$ and $\beta=\frac{1}{2}\left[\left(x_{B}-x_{A}\right) k+\frac{1}{T_{1 A}}-\frac{1}{T_{1 B}}\right]$

$x_{A}, x_{B}$, and $T_{1 A}, T_{1 B}$ being respectively the populations and the longitudinal relaxation times of xenon atoms in the environment $A$ and $B$.

The fit of the curves $I_{A B} / I_{B B}=f\left(t_{m}\right)$ to equation 3 allows us to determine a value of $120 \mathrm{~s}^{-1}$ for the mean exchange rate, $\mathrm{k}$ (Figure 6).

For aluminas Al_237_m and Al_204_m whose porosity is monomodal, the ${ }^{129}$ Xe NMR 1D spectra show only one signal of adsorbed xenon and we studied the exchange between the adsorbed and the gas phase (Figure 7).

For these aluminas we used the signal of the adsorbed phase (signal B) as the reference intensity of diagonal peak rather than that of the gas phase signal (here signal A) considering that the latter is due to all the gas phase atoms situated in the NMR tube but only a fraction can exchange with the adsorbed phase.

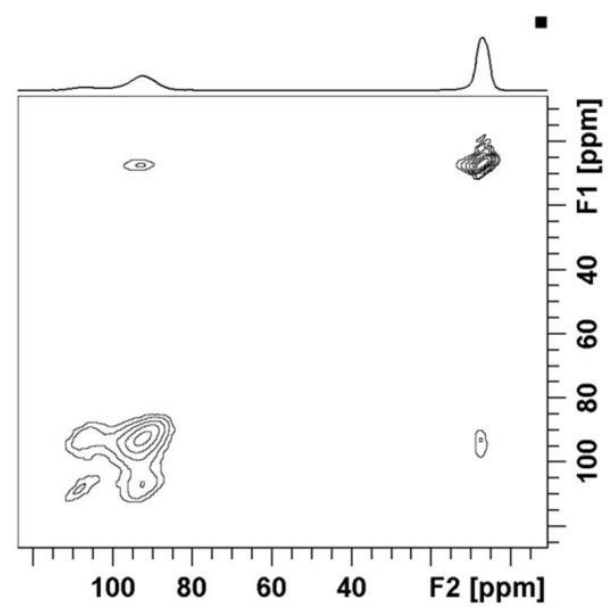

Figure 5. 2D EXSY 129Xe NMR spectrum of xenon adsorbed in Al_224_b for $t_{m}=30 \mathrm{~ms}$.

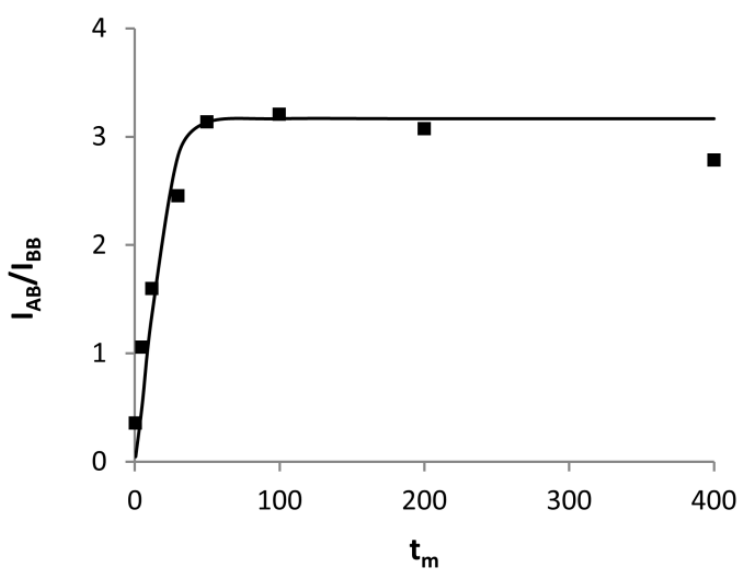

Figure 6. Variation of the IAB/IBB intensity ratio versus the mixing time, tm for xenon exchanging between the porosity A and B of Al_224_b.

The fit of the variations of the ratio $I_{A B} / I_{B B}$ as a function of $t_{m}$ with equation 3 gives $\mathrm{k}$ values of 6 and $130 \mathrm{~s}^{-1}$ for Al_237_m (Figure 8) and Al_204_m respectively. We should keep in mind that these values are underestimated in the case of an exchange of xenon between the porosity and the outer gas phase. Effectively, the population $x_{A}$, used in equation 3 , is the relative intensity of whole the gas phase signal $(A)$ and not only the fraction of gaseous xenon atoms that are able to exchange with the adsorbed atoms.

The reciprocal of the $k$ values corresponds to the average time spent by the atoms in one domain. This residence time, $t_{R}$, is related to the mean square size (radius) of the domain $\left(<R^{2}>\right)$ and to the diffusivity (D) of the atoms in that domain through the equation: ${ }^{6}$

$$
k=\frac{1}{t_{R}}=\frac{15 D}{\left\langle R^{2}\right\rangle}
$$

Equation 4

Taking the diffusivity values measured by ${ }^{129}$ Xe PFG NMR, the mean size of the domain corresponding to the signal considered can be estimated. For the alumina with bimodal porosity (Al_224_b), we can estimate the mean size of each domain (or the mean length of the pores) characterized by a given pore size (6 and $10 \mathrm{~nm})$ using the diffusivities determined by PFG NMR for each types of pores. Using equation 4 we obtained 50 and $70 \mu \mathrm{m}$ for the domains with 6 $\mathrm{nm}$ pores and $10 \mathrm{~nm}$ pores, respectively. These values are rather high and correspond to the size of the agglomerates or even of agglomerates of agglomerates. We can estimate the minimum size of the domains with different porosities by taking into account the fact that there is no coalescence of the signals corresponding to the two types of pores. This means that the exchange between these pores is slower than $830 \mathrm{~Hz}$ (see above). Using equation 4 this implies that the size (radius) of the domains is at least $20 \mu \mathrm{m}$. We can conclude that Al_224_b alumina is constituted of two types of agglomerates, some presenting pores of $6 \mathrm{~nm}$ and others having larger pores $(10 \mathrm{~nm})$. 


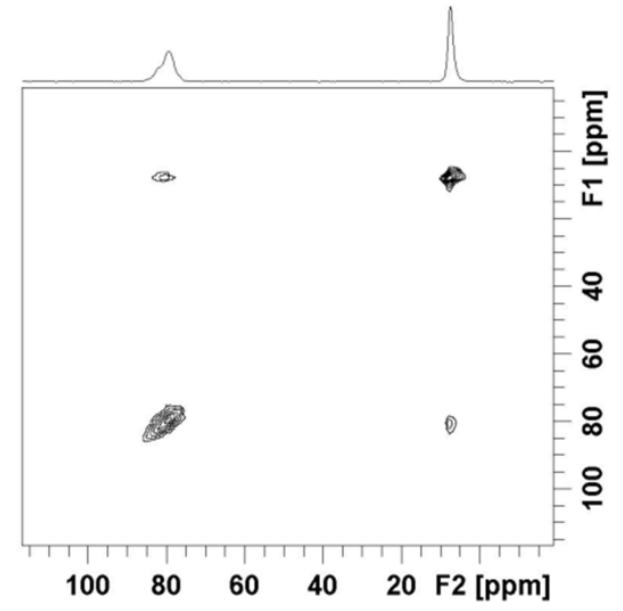

Figure 7. 2D EXSY ${ }^{129}$ Xe NMR spectrum of xenon adsorbed in Al_237_m for tm=50 ms.

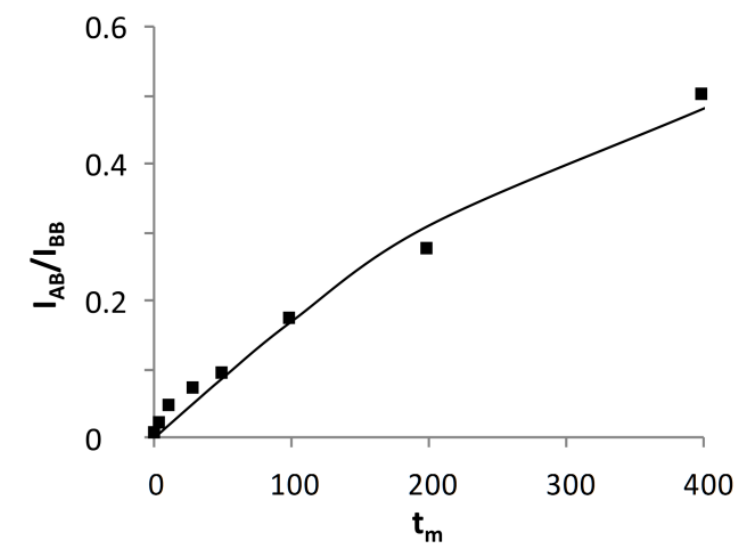

Figure 8. Variation of the $I_{A B} / I_{B B}$ intensity ratio versus the mixing time, $t_{m}$, of xenon exchanging between the adsorbed and the gas phase in $\mathrm{Al}$ _237_m.

For Al_237_m and Al_204_m aluminas having monomodal porosities, we can estimate the size of the porosity domain probed by the xenon atoms. Unfortunately, the diffusivity of xenon in Al_237_m could not be determined because of the too short relaxation times of xenon in this alumina. But if we consider that the diffusivity is similar to that in Al_204_m, we again found large values, around 300 and $80 \mu \mathrm{m}$ for Al_237_m and Al_204_m, respectively. As said above, the exchange rates are underestimated thus, the domain sizes are overestimated and 300 and $80 \mu \mathrm{m}$ are the upper limit values. Nevertheless, the relative errors should be of the same order for the two aluminas and we can conclude that the characteristic porosity arise from agglomerates which are bigger in Al_237_m than in Al_204_m.

\section{Conclusions}

This paper reports ${ }^{1} \mathrm{H}$ and ${ }^{129} \mathrm{Xe}$ PFG NMR results to study the transport properties of $\mathrm{n}$-hexane and xenon within transition alumina pellets used as catalyst supports.

For aluminas impregnated with liquid $\mathrm{n}$-hexane, when the diffusion is governed by molecular interaction (bulk regime) and for adsorbed xenon, when the xenon-pore surface interactions are dominant (Knudsen regime), the measured diffusivity does not depend on the observation time ( $\leq 300 \mathrm{~ms}$ ). This indicates that there are no diffusion barriers and the molecules or atoms diffuse in a homogeneous medium (at a scale of hundreds of micrometres). Therefore, the diffusivity of the diffusing species can be used to evaluate the tortuosity which is a geometric property of alumina porosity. The tortuosities obtained from the diffusivities measured in bulk regime are about ten times smaller than those determined in Knudsen regime. The difference could be attributed to the fraction of molecules or atoms interacting with the pore surface which is more pronounced in the case of adsorbed xenon compared to liquid $\mathrm{n}$-hexane.

In case of aluminas with bimodal porosity, we have shown that, due to the high sensitivity of the xenon electron cloud, ${ }^{129} \mathrm{Xe} \mathrm{NMR}$ is able to differentiate the two types of pores. Consequently, we could determine the diffusivity of xenon and, hence, a tortuosity for each type of pores using ${ }^{129}$ Xe PFG NMR. This demonstrates the power of xenon NMR to study the dynamic and diffusion properties of aluminas presenting complex porosity.

The exchange of xenon between the different pores inside the aluminas and the gas phase has been visualized by $2 \mathrm{D}$ EXSY NMR. Analysis of the 2D spectra allowed probing pore connectivity by evaluating exchange rates of xenon between different pore domains. The combination of these exchange rates with the diffusion coefficients measured by PFG enables us to evaluate the mean size of the alumina pore domains (the order of hundred microns).

\section{References}

1 H. Topsoe and B. S. Clausen, Catal. Rev. - Sci. Eng., 1984, 26, 395-420.

2 J. C. Muijsers, T. Weber, R. M. Vanhardeveld, H. W. Zandbergen and J. W. Niemantsverdriet, J. Catal., 1995, 157, 698-705.

3 H. R. Reinhoudt, A. D. van Langeveld, P. J. Kooyman, R. M. Stockmann, R. Prins, H. W. Zandbergen and J. A. Moulijn, J. Catal., 1998, 179, 443-450.

4 I. E. Maxwell, Catal. Today, 1987, 1, 385-413.

5 D. Li, A. Nishijima and D. E. Morris, J. Catal., 1999, 182, 339-348.

6 J. Kärger and D. M. Ruthven, Diffusion in Zeolites and Other Microporous Solids, John Wiley \& Sons, Inc., New York, 1992.

7 Z. Adem, F. Guenneau, M.-A. Springuel-Huet and A. Gédéon, Microporous Mesoporous Mater., 2008, 114, 337-342.

8 O. Geier, S. Vasenkov, E. Lehmann, J. Karger, U. Schemmert, R. A. Rakoczy and J. Weitkamp, J. Phys. Chem. B, 2001, 105, 10217-10222.

9 F. Stallmach, J. Kärger, C. Krause, M. Jeschke and U. Oberhagemann, J. Am. Chem. Soc., 2000, 122, 9237-9242.

10 Z. Adem, F. Guenneau, M.-A. Springuel-Huet, A. Gédéon, J. lapichella, T. Cacciaguerra and A. Galarneau, J. Phys. Chem. C, 2012, 116, 13749-13759.

11 F. Furtado, P. Galvosas, M. Gonçalves, F.-D. Kopinke, S. Naumov, F. Rodriguez-Reinoso, U. Roland, R. Valiullin and J. Kärger, J. Am. Chem. Soc., 2011, 133, 2437-2443. 
12 A. Menjoge, S. A. Bradley, D. B. Galloway, J. J. Low, S. Prabhakar and S. Vasenkov, Microporous Mesoporous Mater., 2010, 135, 30-36.

13 A. Galarneau, F. Guenneau, A. Gedeon, D. Mereib, J. Rodriguez, F. Fajula and B. Coasne, J. Phys. Chem. C, 2016, 120, 1562-1569.

14 M. Matyka and Z. Koza, ArXiv Prepr. ArXiv12035646, 2012.

15 J. Kärger and S. Vasenkov, Microporous Mesoporous Mater., 2005, 85, 195-206.

16 Jörg Kärger, Douglas M. Ruthven, Doros N. Theodorou, Diffusion in Nanoporous Materials, Weinheim, Germany, Wiley-VCH., 2012.

17 E. Weiland, M.-A. Springuel-Huet, A. Nossov, F. Guenneau, A.-A. Quoineaud and A. Gédéon, J. Phys. Chem. C, 2015, 119, 1528515291.

18 J.-L. Bonardet, J. Fraissard, A. Gédéon and M.-A. Springuel-Huet, Catal. Rev. - Sci. Eng., 1999, 41, 115-225.

19 D. Raftery, Annu. Rep. NMR Spectrosc., 2006, 57, 205-270.

20 E. Weiland, M.-A. Springuel-Huet, A. Nossov and A. Gédéon, Microporous Mesoporous Mater., 2016, 225, 41-65.

21 I. L. Moudrakovski, C. I. Ratcliffe and J. A. Ripmeester, J. Am. Chem. Soc., 1998, 120, 3123-3132.

22 R. M. Cotts, M. J. R. Hoch, T. Sun and J. T. Markert, J. Magn. Reson., 1989, 83, 252-266.

23 E. O. Stejskal and J. E. Tanner, J. Chem. Phys., 1965, 42, 288-292.

24 M. Digne, P. Sautet, P. Raybaud, P. Euzen and H. Toulhoat, J. Catal., 2004, 226, 54-68.

25 P. Nortier, P. Fourre, A. B. M. Saad, O. Saur and J. C. Lavalley, Appl. Catal., 1990, 61, 141-160.

26 P. Euzen, P. Raybaud, X. Krokidis, H. Toulhoat, J.-L. Le Loarer, J.P. Jolivet and C. Froidefond, in Handbook of Porous Solids, eds. F. Schüth, K. S. W. Sing and J. Weitkamp, Wiley-VCH Verlag GmbH, 2002, pp. 1591-1677.

27 N.-K. Bär, S. Ernst, J. Kärger, H. B. Schwarz and J. Weitkamp, Microporous Mater., 1996, 6, 355-361.

28 D. Weber, A. J. Sederman, M. D. Mantle, J. Mitchell and L. F. Gladden, Phys. Chem. Chem. Phys., 2010, 12, 2619-2624.

29 M. Knudsen, J. Membr. Sci., 1995, 100, 23-25.

30 S. Vasenkov and P. Kortunov, Diffus. Fundam., 2005, 1, 2.1-12.

31 C. D'Agostino, J. Mitchell, L. F. Gladden and M. D. Mantle, J. Phys. Chem. C, 2012, 116, 8975-8982.

32 V. N. Burganos, J. Chem. Phys., 1998, 109, 6772-6779.

33 O. Geier, S. Vasenkov and J. Karger, J. Chem. Phys., 2002, 117, 1935-1938.

34 G. K. Papadopoulos, D. N. Theodorou, S. Vasenkov and J. Kärger, J. Chem. Phys., 2007, 126, 094702.

35 J. Demarquay and J. Fraissard, Chem. Phys. Lett., 1987, 136, 314-18.

36 M. Springuel-Huet, J. Demarquay, T. Ito and J. Fraissard, Stud. Surf. Sci. Catal., 1988, 37, 183-9.

37 V. V. Terskikh, I. L. Moudrakovski, S. R. Breeze, S. Lang, C. I. Ratcliffe, J. A. Ripmeester and A. Sayari, Langmuir, 2002, 18, 5653-5656.

38 J. L. Bonardet, A. Gedeon, M. A. Springuel-Huet and J. Fraissard, Mol. Sieves, 2007, 5, 155-248.

39 J. Jeener, B. H. Meier, P. Bachmann and R. R. Ernst, J. Chem. Phys., 1979, 71, 4546-4553. 